\title{
Growth Habits of Polymer Single Crystals
}

\author{
Shizuo HAYASHI \\ Department of Physics, Faculty of General Study, Gunma University, \\ Aramaki-cho Maebashi, Japan.
}

(Received July 4, 1974)

\begin{abstract}
The uniform and definite thickness of polymer single crystals is explained by introducing the concept of the excess free energy preventing the indefinite increase of thickness. The excess free energy is proved to be proportional to the square of thickness from the viewpoint of the crystal defect concept. The free energy contour diagram of crystal growth has a saddle point and a ravine parallel to the width axis. The value of the height axis corresponding to the bottom of the ravine is the thickness of polymer single crystal, which does not exceed a definite value. The ravine shifts upward along the height axis and thickness increases with elevating the crystallization temperature. The thickening rate by heat treatment is obtained by the method of the thermodynamics of irreversible processes, the thickness increases linearly in the earlier stage and tends in the final stage to a finite value characteristic of the heat treatment temperature.
\end{abstract}

KEY WORDS Single Crystal / Crystal Growth / Crystal Defect / Contour Diagram / Thickening / Heat Treatment /

Until the discovery of polymer single crystals, ${ }^{1}$ it was considered that polymeric substances do not crystallize perfectly and some amorphous part remained. The striking phenomenon with plate-like polymer single crystals is their finite and uniform thickness of about $100 \AA$ in the chain direction and the inverse temperature dependence of their thickness. ${ }^{1}$ These results were interpreted from the view point of kinetic theory. ${ }^{2,3}$

The theories already presented are not considered to be satisfactory. One point is concerned with the shape of the free energy contour diagram of crystal growth. When the crystal growth is considered to be controlled by the nucleation of a monolayer of polymer chains, having width $a$, height $b$, and thickness $d$, added on the lateral plane of the crystal, the free energy $\Delta F$ required to form this size of monolayer satisfies the relation ${ }^{2,3}$

$$
\left(a-a^{*}\right)\left(b-b^{*}\right)=-\left(\Delta F-\Delta F^{*}\right) / d \Delta G
$$

where $\Delta G$ is the free energy difference between liquid and crystal phases, $a^{*}, b^{*}$, and $\Delta F^{*}$ are the respective values of $a, b$, and $\Delta F$ corresponding to the critical size of the monolayer nucleus.
The contour lines at fixed values of $\Delta F$ are right hyperbola whose asymptotes cross at the point $\left(a^{*}, b^{*}\right)$ and are parallel to the $a$ - and $b$ axes and the free energy surface is saddle-shaped with the saddle point at $\left(a^{*}, b^{*}, \Delta F^{*}\right)$ as shown in Figure 1 where the chain direction is chosen in the $b$-axis.

In explaining the formation of plate-like crystals with folded chains, the condition that $b$ is fixed at a value nearly equal to $b^{*}$ is assumed by using the reason that $b$ does not increase owing to the folded structure of the chains but judging from the contour diagram of crystal growth (Figure 1) $b$ must increase as the monolayer of chains grows larger along on the lateral plane.

The other point is the shape of the crystal in the equilibrium state. When a single crystal is annealed after the termination of crystal growth, the chains in the crystal are expected to transform relatively easily to the thermodynamically stable configuration since the thickening of the crystal by heat treatment is easily realized.

When the surface free energies of loop and lateral planes are denoted by $\sigma_{\theta}$ and $\sigma_{\mathrm{s}}$, the ratio of thickness to width at the stable state 


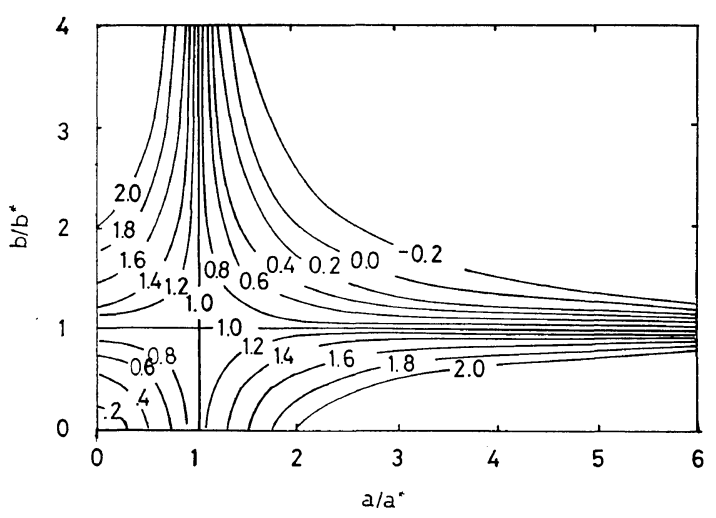

Figure 1. Free energy contour diagram of crystal growth at fixed $\Delta F$ predicted from eq 1 . When the monolayer grows larger on the lateral surface, the height must increase indefinitely as the growth of monolayer.

is predicted to be $\sigma_{\mathrm{e}} / \sigma_{\mathrm{s}}(\sin \theta)^{1 / 2}, \theta$ being the angle between the sides in the rhombic plane of the single crystal. As $\sigma_{\mathrm{e}}$ is far larger than $\sigma_{\mathrm{s}}$, the expected shape of crystal is one of a rectangular prism with a large height and a small base. However, the experimental shape differs from the above expectation; the plate-like shape is maintained during the heat treatment process though the thickness increases as the heat treatment temperature is elevated. Therefore, we should consider some structural effects of crystal as preventing the indefinite increase of thickness in the crystallization process. It is also expected that these effects may have some influence on the shape of the contour diagram of crystal growth.

As a kind of structural effect we consider a sort of crystal defect or crystal imperfection formed in the process of crystal growth. The arrangement of the units of the polymer molecules in the thickness direction generally may not be perfect according to the characteristic properties of polymer molecules in that each unit is joined to the neighbouring one and each unit in chains cannot always occupy the lowest freee energy state. However, some of the units will be frozen in higher free energy states and thus the crystal may have excess free energy resulting in crystal defects or crystal imperfections.

Taking into account the effect of crystal defects we investigate the crystal growth and some properties of the polymer single crystal.

\section{FREE ENERGY OF THE CRYSTALLITE}

We consider a single crystal composed of $p^{2}$ chains each containing $q$ units, $p$ corresponds to the width and $q$ to the thickness, and the unit is taken as $\mathrm{CH}_{2}$ for polyethylene. The crystal of this size is termed the $p-q$ crystal for the sake of simplicity. The thickness of crystal is measured by the number of units in a chain and the width by the number of chains in a side of rhombic plane of the crystal.

Let the surface free energy per loop of the end plane be $\varepsilon_{\mathrm{e}}$, $\varepsilon_{\mathrm{s}}$ that per unit of the lateral plane and $\varepsilon_{\mathrm{f}}$ the free energy difference per unit between solid and liquid phases. The free energy of forming the $p-q$ crystal in the absence of excess free energy induced by the crystal defect is then expressed as

$$
\Delta \phi^{\prime}=2 p^{2} \varepsilon_{\mathrm{e}}+4 p q \varepsilon_{\mathrm{s}}-p^{2} q \varepsilon_{\mathrm{f}}
$$

These quantities $\varepsilon_{\mathrm{e}}, \varepsilon_{\mathrm{s}}$, and $\varepsilon_{\mathrm{f}}$ are related to the surface free energy of the end plane $\sigma_{\mathrm{e}}$, that of the lateral plane $\sigma_{\mathrm{s}}$ and the free energy of crystallization $\Delta G$ by $\varepsilon_{\mathrm{e}}=\sigma_{\mathrm{e}} l^{2} \sin \theta, \varepsilon_{\mathrm{s}}=\sigma_{\mathrm{s}} k l$ and $\varepsilon_{\mathrm{f}}=\Delta G k l^{2} \sin \theta, l$ being the distance between two neighbouring chains and $k$ is the length of the unit.

Next we consider the excess free energy which prevents the indefinite increase of crystal thickness. This is considered to originate in the crystal defect mentioned in the previous section. Since any term proportional to the thickness must be included in the free energy of crystallization as a part, the power dependence of the excess free energy on thickness must be higher than unity. Here we assume that the excess free energy is proportional to the square of thickness. Then the free energy of forming the $p-q$ crystal is

$$
\Delta \phi=2 p^{2} \varepsilon_{\mathrm{e}}+4 p q \varepsilon_{\mathrm{s}}-p^{2} q \varepsilon_{\mathrm{f}}+\lambda p^{2} q^{2}
$$

where $\lambda$ is the proportionality constant depending on the crystallization temperature and $\lambda q^{2}$ is the excess free energy per chain. ${ }^{4-6}$ One method of obtaining the last term of eq 3 is given in Section 5.

\section{PRIMARY NUCLEATION}

When we consider the primary nucleation, 
the free energy of forming the $p-q$ size nucleus is also expressed as eq 3. Minimizing $\Delta \phi$ we obtain the critical size of the primary nucleus as

$$
\begin{gathered}
q^{*}=4 \varepsilon_{\mathrm{e}} / \varepsilon_{\mathrm{f}} \\
p^{*}=4 \varepsilon_{\mathrm{s}} / \varepsilon_{\mathrm{f}}\left(1-8 \lambda \varepsilon_{\mathrm{e}} / \varepsilon_{\mathrm{f}}{ }^{2}\right)
\end{gathered}
$$

When $p$ and $q$ exceed the critical values, a crystal begins to grow. The critical value $p^{*}$ is larger by the factor $\left(1-8 \lambda \varepsilon_{\mathrm{e}} / \varepsilon_{\mathrm{f}}^{2}\right)^{-1}$ than that obtained from eq 2 ; the critical size of the primary nucleus predicted from eq 3 is larger in width than that from eq 2 . The critical free energy of forming the nucleus is given as

$$
\Delta \phi^{*}=32 \varepsilon_{\mathrm{s}}{ }^{2} \varepsilon_{\mathrm{e}} / \varepsilon_{\mathrm{f}}{ }^{2}\left(1-8 \lambda \varepsilon_{\mathrm{e}} / \varepsilon_{\mathrm{f}}{ }^{2}\right)
$$

If $\lambda$ is large enough, $p^{*}$ becomes negative and the nucleation cannot occur, but in the present treatment $\lambda$ is not so large that $p^{*}$ does not have a negative value. When the value of $\lambda$ tends to zero the above results agree with those of Lauritzen and Hoffman ${ }^{3}$ and of Price. ${ }^{2}$

\section{CONTOUR DIAGRAM OF CRYSTAL GROWTH}

Taking the excess free energy into account, we investigate the crystal growth. We consider that the crystal growth is brought about by the addition of new chains on the lateral plane subsequent to the primary nucleus formation. The model for forming the secondary nucleus is the ordinary one as shown in Figure 2.

A new monolayer composed of $t$ chains, each containing $h$ units, is added on the lateral surface and the secondary nucleus is formed. The free energy required to form this nucleus from the liquid phase is

$$
\Delta \phi_{\mathrm{g}}=2 t \varepsilon_{\mathrm{e}}+2 h \varepsilon_{\mathrm{s}}-t h \varepsilon_{\mathrm{f}}+\lambda t h^{2}
$$

The criteria of forming the secondary nucleus

$$
\left.\begin{array}{l}
\partial \Delta \phi_{\mathrm{g}} / \partial t=0 \\
\partial \Delta \phi_{\mathrm{g}} / \partial h=0
\end{array}\right\}
$$

give

$$
h_{1}^{*}=\varepsilon_{\mathrm{f}}\left(1-\left[1-8 \lambda \varepsilon_{\mathrm{e}} / \varepsilon_{\mathrm{f}}^{2}\right]^{1 / 2}\right) / 2 \lambda
$$

and

$$
t^{*}=2 \varepsilon_{\mathrm{s}} / \varepsilon_{\mathrm{f}}\left(1-8 \lambda \varepsilon_{\mathrm{e}} / \dot{\varepsilon}_{\mathrm{f}}^{2}\right)^{1 / 2}
$$

respectively. The critical free energy of forming the secondary nucleus is

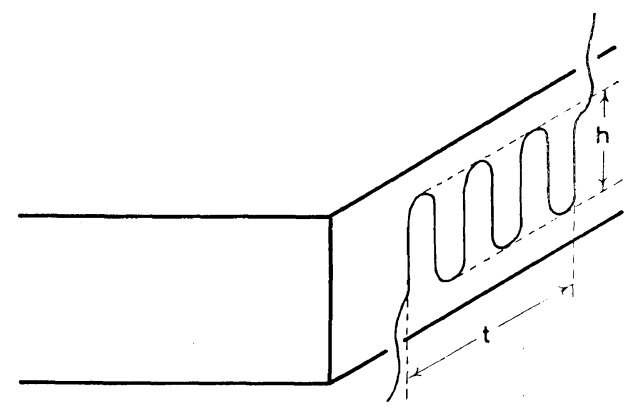

Figure 2. Addition of new monolayer of polymer molecules on the lateral surface. The monolayer contains $t$ chains each having $h$ units.

$$
\Delta \phi_{\mathrm{g}}{ }^{*}=\frac{\varepsilon_{\mathrm{s}} \varepsilon_{\mathrm{f}}}{\lambda}\left(1-\left[1-8 \lambda \varepsilon_{\mathrm{e}} / \varepsilon_{\mathrm{f}}\right]^{1 / 2}\right)
$$

The condition of eq 8 gives a quadratic equation of $h$ and the other root of $h$ from the equation is

$$
h_{2}^{*}=\varepsilon_{\mathrm{f}}\left(1+\left[1-8 \lambda \varepsilon_{\mathrm{e}} / \varepsilon_{\mathrm{f}}{ }^{2}\right]^{1 / 2}\right) / 2 \lambda
$$

but the critical free energy of forming the nucleus corresponding to $h_{2}{ }^{*}$ is larger than $\Delta \phi_{\mathrm{g}}{ }^{*}$ and at the same time $h_{2}{ }^{*}>2 q^{*}$, therefore the chance of forming the latter nucleus having $h_{2}{ }^{*}$ is far less than the former.

When we introduce the following variables and parameters

$$
\begin{aligned}
& t / t^{*}=\tau, \quad h / h_{1}^{*}=x \\
& \left.\left(h_{2}{ }^{*}-h_{1}{ }^{*}\right) / h_{1}{ }^{*}=\eta, \quad \Delta \phi_{\mathrm{g}} / \Delta \phi_{\mathrm{g}}{ }^{*}=\phi\right\}
\end{aligned}
$$

we have

$$
\tau=\eta\left\{\frac{\phi-x}{(x-1)(x-1-\eta)}\right\}
$$

This gives the $t v s$. $h$ relation for each fixed value of $\phi$. Taking the parameter $\eta$ as 2 we have the free energy contour diagram of crystal growth shown in Figure 3. This contour diagram has a saddle point $\mathrm{A}$ at $\left(h_{1}{ }^{*}, t^{*}, \Delta{\phi_{\mathrm{g}}}^{*}\right)$ and a ravine $\mathrm{B}$ parallel to the $t$-axis, accordingly, a crystal may grow along the gradient of the contour diagram to the bottom of the ravine passing the neighbourhood of the saddle point.

Thus, as the crystal grows, its thickness attains the value of the $h$-axis corresponding to the bottom of the ravine but can not exceed this value irrespective of the intermediate process of crystallization. When $t$ is large this value is given as 


\section{S. HAYASHI}

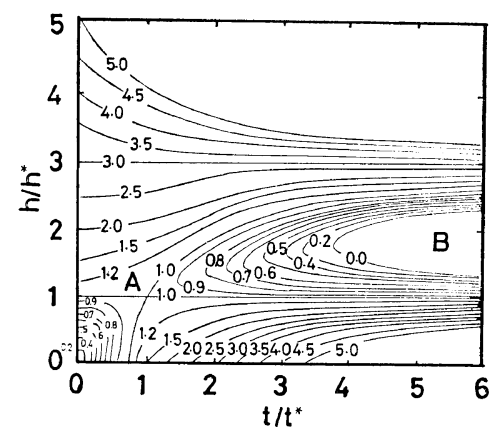

Figure 3. Free energy contour diagram of crystal growth at fixed $\phi$ predicted from eq 14. The number shows the value of $\phi$. The height of the added monolayer has the value of $h$ axis corresponding to the bottom of the ravine $B$, and the thickness of the single crystal has a definite value irrespective of the size.

$$
h^{*}=\left(h_{1}{ }^{*}+h_{2}{ }^{*}\right) / 2=\varepsilon_{\mathrm{f}} / 2 \lambda
$$

As $\eta$ increases ( $\lambda$ decreases) the line indicated as 1.0 transfer to the straight line parallel to the $h$-axis; the contour lines at fixed $\phi$ transform to the right hyperbola as $\lambda$ tends to zero, in this case, $h^{*}$ tends to infinity as the crystal grows larger and larger.

\section{THICKNESS IN STABLE STATE}

The stable state in the present consideration is the quasi-equilibrium state in which the crystal defect is frozen in, therefore the thickness in the stable state means the quasi-equilibrium thickness. This thickness is obtained by minimizing $\Delta \phi$ in eq 3 at constant temperature and constant volume. Then, for large crystals with large $p$ we have

$$
q^{\prime}=\left(2 \varepsilon_{\mathrm{e}} / \lambda\right)^{1 / 2}
$$

where the higher order terms than $q / p$ are neglected since $q$ is smaller than a definite value irrespective of $p$.

Since we have

$$
h^{*} q^{*}=q^{\prime 2}
$$

from eq 4, 15, and 16, we see that $q^{\prime}$ has a value between $q^{*}$ and $h^{*}$. Therefore, in the fully grown or matured crystal, the thickness $q^{\prime}$ does not exceed a finite value determined by the crystallization temperature no matter what the size of the crystal is.
In the present consideration $q^{\prime}$ corresponds to the experimentally measured crystal thickness, the dependence of which on the crystallization temperature is inversely proportional to the degree of supercooling $\Delta T$, where $\Delta T=T_{\mathrm{m}}-T_{\mathrm{c}}, T_{\mathrm{m}}$ is the melting temperature of crystal and $T_{\mathrm{c}}$ is the crystallization temperature. Therefore, $q^{\prime} \propto 1 / \Delta T$ and $\lambda$ is expected to be proportional to $(\Delta T){ }^{2}$ Whether the temperature dependence of $\lambda$ is obtained by using the crystal defect concept is examined in the next section.

\section{AN INTERPRETATION OF EXCESS FREE ENERGY TERM}

We investigate a method of deriving the excess free energy term, $\lambda q^{2}$, from the viewpoint of crystal defect.

If there is no crystal defect, a chain in a crystal may form a trans zig-zag plane and all the units in a chain occupy the lowest energy state. However, in the actual process of crystal growth, all the units in a chain may not always be in the lowest energy state and some of them will be in higher one. In other words, all the units in a chain may not always be arranged in the trans conformation and some of them will take the gauche position.

We consider such a model of chain in a crystal; the chain contains $n$ subgroups of units lining up on a zig-zag plane, the position connecting two neighbouring subgroups of units have a complex arrangement of a few units including the mixed trans-and-gauche conformation, this position may be considered a sort of point dislocation, the defect point.

The zig-zag planes of subgroups will not in general be on the same plane owing to the effect of the defect points. We choose a reference plane which has the lowest free energy in its arrangement of units. Each zig-zag plane of subgroup of units will have some deviation from the reference plane, this deviation will induce the excess free energy of a chain.

Let us consider a chain interacting with its surrounding chains. For the sake of simplicity, the intermolecular interaction is approximated by an average potential field produced by the surrounding chains. The potential energy of the reference plane is zero. When the zig-zag 
plane of the $i$-th subgroup of units is simply termed "the $i$-th plane," it has a free energy increase determined by its deviation from the reference plane. This free energy increase may be approximated by the energy increase in the average potential field mentioned above. Then, if the deviation angle of the $i$-th plane is denoted as $\theta_{i}$, the free energy increase of the $i$-th plane should be expressed as eq 18 as a first approximation

$$
f_{i}=\kappa\left\langle\theta_{i}{ }^{2}\right\rangle+w
$$

where $\kappa$ is a constant having similar meaning to distortion modulus, $w$ is the free energy increase per defect point and \langle\rangle denotes the average with respect to chain conformation. The deviation of each plane may occur nearly randomly in both the positive and negative directions from the reference plane by a factor $\Delta \theta, \Delta \theta$ being the deviation angle per defect point, $\left\langle\theta_{i}{ }^{2}\right\rangle$ is expected to be proportional to $i \Delta \theta^{2}$ and eq 18 becomes

$$
f_{i}=\kappa i \Delta \theta^{2}+w
$$

Summing up eq 19 we have the excess free energy

$$
f(\lambda)=\sum_{i=1}^{n} f_{i} \simeq n^{2} \kappa \Delta \theta^{2} / 2+n w
$$

and the next question is to evaluate $n$.

The density of crystal defects may be smaller when a crystal grows slowly at a high temperature than when it grows rapidly at a low temperature. Though the defect points may be formed in the process as each unit is lined up step by step on the lateral plane, the defect point may have a chance to be removed after the lining-up of units. The number of defect points initially formed in the process of crystallization at high temperature will be large because of the violent thermal motion at high temperature. However, the chance of the initially formed defect point disappearing will be also large at high temperature and superior to the chance of forming the defect point initially. Accordingly, the density of the finally frozen defect points in a crystal will be small at high temperatures and large at low temperatures and it will be controlled mainly by the disappearance of crystal defects. The concept of crystal defect mentioned in our treatment is concerned with the frozen defect and the defect point means the frozen one.

Let the probability of forming a frozen defect point at a temperature $T$ be $P(T)$, then $q P(T)$ defect points are formed in a chain. Here, $P(T)$ is defined in the meaning of the quasiequilibrium state. The number of subgroups of units in a chain $n$ is equal to $q P(T)$ in our approximation and eq 20 becomes

$$
f(\lambda)=q^{2} \kappa \Delta \theta^{2} P(T)^{2} / 2+q w P(T)
$$

The second term proportional to $q$ in eq 21 should be the term included in $\varepsilon_{\mathrm{f}}$, therefore the excess free energy term of a chain must be equal to the first term of eq 21 , and we have

$$
\lambda=\kappa \Delta \theta^{2} P(T)^{2} / 2
$$

Since $P(T)$ should be controlled by the whole kinetic process of crystal growth, the practical evaluation of $P(T)$ is very difficult. However, when we want to know only the temperature dependence of $P(T)$, it will be obtained qualitatively by a phenomenological consideration. Let the melting temperature of a large crystal without any crystal defect be $T_{\mathrm{m}}$, the equilibrium melting and recrystallization taking place at $T_{\mathrm{m}}$ under constant pressure. Then, if a crystal were formed at $T_{\mathrm{m}}$, it should have no crystal defect, that is $P\left(T_{\mathrm{m}}\right)=0$. When the degree of supercooling is not so large, $P(T)$ may be approximated by the first order term of supercooling as

$$
P(T)=A \Delta T / T_{\mathrm{m}}
$$

where $\Delta T / T_{\mathrm{m}}$ is the degree of supercooling given by $\left(T_{\mathrm{m}}-T\right) / T_{\mathrm{m}}, T$ being the crystallization temperature, $A$ is a constant.

By the use of eq 23, eq 22 leads to

$$
\lambda=\frac{A^{2} \kappa \Delta \theta^{2}}{2}\left(\frac{\Delta T}{T_{\mathrm{m}}}\right)^{2}
$$

This is the temperature dependence of $\lambda$.

The crystal thickness is nearly equal to the quasi-equilibrium thickness $q^{\prime}$ given by eq 16 , we have

$$
q^{\prime}=\frac{2}{A \Delta \theta}\left(\frac{\varepsilon_{\mathrm{e}}}{\kappa}\right)^{1 / 2}\left(\frac{T_{\mathrm{m}}}{\Delta T}\right)
$$

This is the well known crystallization tempera- 


\section{S. HAYASHI}

ture vs. thickness relation already discussed in Section 4.

The temperature dependence of $\lambda, \lambda \propto(\Delta T)^{2}$, discussed in Section 4 is obtained by using the crystal defect model in this section.

\section{RATE OF THICKENING BY HEAT TREATMENT}

The thickening rate of a polyethylene single crystal by heat treatment was measured by observing the long period pattern of narrow Xray diffraction ${ }^{7-9}$ and the experimental thickness $v s$. time relation was found for the long time scale to be

$$
q \propto \ln t
$$

This relation is explained by Hirai, et al. ${ }^{9}$ by using the method of a rate process, but this shows that the thickness increases indefinitely with time. However, the recent work for single crystals formed from monodispersed polyethylene shows that the thickness of single crystal seems to reach a maximum at a finite value characteristic of the heat treatment temperature and the molecular weight. ${ }^{10,11 *}$

When a crystal is treated by heating at an elevated temperature, the crystal defect may be decreased by the same process of disappearance of the defect points as mentioned in Section 5. Though the disappearance way in which the defect points disappear in the process of annealing may generally differ from that in the process of crystallization, the density of crystal defect should be controlled by the disappearance of the defect points as already mentioned in the previous section. In addition, in our approximation, the relation between $\lambda$ and temperature which is derived by using the temperature dependence of the probability of forming the frozen defect points may be applicable to the case of thickening by heat treatment. We then use eq 24 for $\lambda$ in the qualitative treatment of the rate of thickening by heat treatment.

When the temperature is elevated from a initial temperature $T_{0}$ to an annealing temperature $T_{\mathrm{a}}$, the thickness $q$ increases from the initial-

* Properties affected by molecular weight are outside of the consideration in the present treatment and will be treated in another article. value $\left[2 \varepsilon_{\mathrm{e}} / \lambda\left(T_{0}\right)\right]^{1 / 2}$ to the final one $\left[2 \varepsilon_{\mathrm{e}} / \lambda\left(T_{\mathrm{a}}\right)\right]^{1 / 2}$ by our theory, where $\lambda\left(T_{0}\right)>\lambda\left(T_{\mathrm{a}}\right)$. Once the defect is diminished by heat treatment, the crystal should not return to the initial defectrich state even though it is cooled down again, since the defect-poor state is more stable. The thickening by heat treatment is therefore expected to be irreversible.

We formulate the above consideration of thickening by heat treatment on the basis of the thermodynamics of irreversible processes. The driving force or affinity $X$ inducing the thickening of the crystal is given by using eq 3

$$
X=-\left(\frac{\partial \Delta \phi}{\partial q}\right)_{T, N}=N\left(\frac{2 \varepsilon_{\mathrm{e}}}{q^{2}}-\lambda\right)
$$

for a large crystal where $N=p^{2} q$. By analogy with other irreversible processes the phenomenological law for the thickening rate is written as

$$
\frac{\mathrm{d} q}{\mathrm{~d} t}=L X=L N\left(\frac{2 \varepsilon_{\mathrm{e}}}{q^{2}}-\lambda\right)
$$

$L$ is the proportionality constant. In the case of quasi-equilibrium state, $\mathrm{d} q / \mathrm{d} t=0$, and eq 16 is obtained again for the thickness which corresponds to the value of $q$ at infinite $t$, namely $q_{\infty}=\left(2 \varepsilon_{\mathrm{e}} / \lambda\right)^{1 / 2}$. Writing $q_{0}$ for the initial value of $q$, we obtain the $q$ vs. $t$ relation from eq 27 ,

$$
\begin{aligned}
\frac{q}{q_{\infty}} & +\frac{1}{2} \ln \frac{q_{\infty}-q}{q_{\infty}+q} \\
& =\frac{q_{0}}{q_{\infty}}+\frac{1}{2} \ln \frac{q_{\infty}-q_{0}}{q_{\infty}+q_{0}}-\frac{2 L N \varepsilon_{\mathrm{e}}}{q_{\infty}{ }^{3}} t
\end{aligned}
$$

The thickening behavior predicted from eq 28 in short and long time ranges are

$$
\begin{aligned}
& q \simeq q_{0}+\frac{q_{\infty}}{2 q_{0}{ }^{2}}\left(q_{\infty}{ }^{2}-q_{0}{ }^{2}\right) A t, \\
& \text { for short time ranges } \\
& q \simeq q_{\infty}\left(1-2 \frac{q_{\infty}-q_{0}}{q_{\infty}+q_{0}} e^{-\left(2\left(q_{\infty}-q_{0}\right) / q_{\infty}\right)} e^{A t}\right), \\
& \text { for long time ranges } \\
& A \equiv 4 L N \varepsilon_{\mathrm{e}} / q_{\infty}{ }^{3}
\end{aligned}
$$

namely, the thickness increases linearly with time in the earlier stage and tends to a finite value in the final stage. This tendency qualitatively agrees with the behavior observed experimentally. 
In the above treatment $\lambda$ is assumed not to depend on time and to take a finite value $\lambda\left(T_{\mathrm{a}}\right)$. However, $\lambda$ may generally depend on time and may be expressed as

$$
\left.\begin{array}{l}
\lambda(t)=\lambda_{\infty}+\left(\lambda_{0}-\lambda_{\infty}\right) \varphi(t) \\
\varphi(t)=1, \text { for } t=0 \\
0<\varphi(t)<1, \text { for } 0<t<\infty \\
\varphi(t)=0, \text { for } t=\infty
\end{array}\right\}
$$

where $\lambda_{0}=\lambda\left(T_{0}\right)$ and $\lambda_{\infty}=\lambda\left(T_{\mathrm{a}}\right)$. In order to facilitate the calculation, we choose a formula for $\lambda(t)$;

$$
\lambda(t)=\lambda_{\infty}+\left(\lambda_{0}-\lambda_{\infty}\right) \frac{q_{0}{ }^{2}}{q_{\infty}{ }^{2}-q_{0}{ }^{2}} \frac{q_{\infty}{ }^{2}-q^{2}}{q^{2}} \psi(t)
$$

which may be equivalent to eq 30 , where $\psi(t)$ have the same properties as $\varphi(t)$ in eq 30 . Then we obtain from eq 27

$$
\begin{aligned}
\frac{q}{q_{\infty}} & +\frac{1}{2} \ln \frac{q_{\infty}-q}{q_{\infty}+q} \\
& =\frac{q_{0}}{q_{\infty}}+\frac{1}{2} \ln \frac{q_{\infty}-q_{0}}{q_{\infty}+q_{0}}-\frac{2 L N \varepsilon_{\mathrm{e}}}{q_{\infty}^{3}}\left(t-\int_{0}^{t} \psi\left(t^{\prime}\right) \mathrm{d} t^{\prime}\right)
\end{aligned}
$$

which gives a slower rate of thickening than that predicted from eq 28.

\section{SUMMARY AND DISCUSSION}

Introducing the excess free energy term we obtained the free energy contour diagram of crystal growth, which has a narrow ravine parallel to the width axis. The crystal grows along the gradient of the contour diagram to the bottom of the ravine, namely, the fully grown crystal has a plate-like shape with a uniform and definite thickness irrespective of the size of the crystal. Therefore, we obtain an interpretation as to the plate-like polymer single crystal is formed by using the excess free energy term.

Owing to the present consideration, the critical condition giving rise to the chain folding is controlled by the balance between the fold-free energy of chains and the excess free energy. Since the excess free energy increases with the second power of thickness, it may overstep the fold-free energy of the chains when the thickness exceeds a critical value. When a crystal be- comes thicker than the critical value it has higher free energy than the thinner crystal with a folded structure consequently, the crystal with folded chains, having a plate-like shape and definite thickness thinner than the critical value, can survive as a stable crystal.

We can also explain the irreversible thickening by heat treatment. When the temperature is elevated slowly, the crystal defect may be reduced by the thermal motion of units in chains and the excess free energy will be reduced, consequently, the thickness increases with raising the heat treatment temperature. This thickening phenomena is irreversible since the crystal may not return to the former defect-rich state even though it is cooled down.

In the present work, the excess free energy term is considered to originate from the frozen crystal defects. The reason for reaching this consideration is to explain the irreversible thickening by heat treatment. If this term is related to the lattice vibration, the thickening must be reversible.

Although we obtain the excess free energy term from the model of Section 5, this model is not the only one. Another model ${ }^{5}$ also leads to the same result, the square dependence on the thickness, but, does not the temperature dependence of $\lambda$. Accordingly, the present model is considered to be one of the best for the excess free energy term. However the direct certification of this model may be impossible. The existence of the excess free energy term may be ascertained by obtaining the theoretical results capable of determining a parameter, such as $\lambda q^{2}$, experimentally. The results, perhaps, contain the apparent melting temperature.

\section{REFERENCES}

1. A. Keller and A. O'Conner, Discuss. Faraday Soc., 25, 114 (1958).

2. J. I. Lauritzen and J. D. Hoffman, J. Res. Nat. Bur. Stand., A, 64, 73 (1960).

3. F. P. Price, J. Polym. Sci., 42, 49 (1960).

4. Hayashi, T. Takizawa, Y. Takano, and C. Shibuya, Rep. Progr. Polym. Phys. Japan, 11, 107 (1968).

5. S. Hayashi and T. Takizawa, ibid., 14, 107 (1971).

6. S. Hayashi and T. Takizawa, J. Cryst. Growth, 
24/25, 591 (1974).

7. W. O. Statton and P. H. Geil, J. Appl. Polym. Sci., 3, 357 (1960).

8. E. W. Fischer and G. F. Schmit, Angew. Chem., 74, 551 (1962).

9. N. Hirai, T. Mitsuhata, and H. Yamashita,
Kobunshi Kagaku (Chem. High Polymers), 18, 33 (1961).

10. M. Takayanagi and F. Nagatoshi, Mem. Fac. Eng. Kyushu Univ., 24, 33 (1965).

11. E. W. Fisher, Kollod.-Z. Z. Polym., 231, 458 (1969). 